\title{
Generalized approach to quantify correlations in bipartite quantum systems
}

\author{
D. G. Bussandri ${ }^{1,2}$, A. P. Majtey ${ }^{1,3}$, P. W. Lamberti1 ${ }^{1,2}$, T. M. Osán ${ }^{1,3}$ \\ ${ }^{1}$ Facultad de Matemática, Astronomía, Física y Computación, \\ Universidad Nacional de Córdoba, Av. Medina Allende s/n, \\ Ciudad Universitaria, X5000HUA Córdoba, Argentina \\ ${ }^{2}$ Consejo Nacional de Investigaciones Científicas y Técnicas de la República Argentina, \\ Av. Rivadavia 1917, C1033AAJ, CABA, Argentina and \\ 3 Instituto de Física Enrique Gaviola, \\ Consejo Nacional de Investigaciones Cientificas y Técnicas de la República Argentina, \\ Av. Medina Allende s/n, X5000HUA, Cŕdoba, Argentina
}

\begin{abstract}
In this work we developed a general approach to the problem of detecting and quantifying different types of correlations in bipartite quantum systems. Our method is based on the use of distances between quantum states and processes. We rely upon the premise that total correlations can be separated into classical and quantum contributions due to their different nature. In addition, according to recently discussed criteria, we determined the requirements to be satisfied by distances in order to generate correlation measures physically well-behaved. The proposed measures allow us to quantify quantum, classical and total correlations. Besides the well-known case of relative entropy we introduce some additional examples of distances which can be used to build bona fide quantifiers of correlations.
\end{abstract}

\section{INTRODUCTION}

Quantum information processing and quantum computing are relatively new subjects of research concerned with the use of quantum resources to perform tasks of information processing which are either not feasible to be implemented classically or can be performed with classical devices in a much less efficient way. Since the inception of these research fields, the central point seems to be able to identify which features in the quantum realm are responsible for the so-called quantum advantage. Among the possible quantum features, entanglement 
is regarded as the main resource for practical use in quantum information processing [1 14$]$, particularly, for quantum protocols in quantum networks (composite systems). However, at present it is widely recognized that this is not the unique asset which can be used for quantum protocols in order to outperform their classical counterparts. Several results allow us to conclude that, in some quantum tasks, correlations of a quantum nature different from entanglement are responsible for the processing improvements [15-22]. In fact, different works on quantum correlations have been devoted to analyze their resilience to noise under certain conditions [23 27]. Also, it is worth mentioning that quantum nonlocality may also arise even in the absence of entanglement [28].

Usually, because of the distinct nature of quantum and classical correlations [29 32], the procedures used to detect the presence of correlations rely upon the reasonable hypothesis that total correlations $\mathcal{T}$ contained in a bipartite quantum state $\rho$ can be quantified as follows

$$
\mathcal{T}(\rho)=\mathcal{Q}(\rho)+\mathcal{J}(\rho)
$$

where $\mathcal{Q}$ and $\mathcal{J}$ represent measures of quantum and classical correlations, respectively. It is important to realize that, within this framework, Eq. (1) makes sense if all three quantities are measured in the same units. In particular, a widely accepted information-theoretic measure of total correlations contained in a bipartite quantum state $\rho$ is (von Neumann) Quantum Mutual Information $\mathcal{I}(\rho)$ defined as:

$$
\mathcal{I}(\rho) \doteq S\left(\rho_{A}\right)+S\left(\rho_{B}\right)-S(\rho)
$$

In Eq. (2), $\rho$ stands for a general bipartite quantum state, $\rho_{A}=\operatorname{Tr}_{B}\left[\rho_{A B}\right]$ and $\rho_{B}=$ $\operatorname{Tr}_{A}\left[\rho_{A B}\right]$ represent the corresponding reduced (marginal) states, and $S(\rho)$ represents the von Neumann entropy given by

$$
S(\rho)=-\operatorname{Tr}\left[\rho \log _{2} \rho\right]
$$

The quantity $\mathcal{I}(\rho)$ describes the correlations between the whole subsystems rather than a correlation between just two observables.

Classical correlations contained in a quantum state $\rho$ of a bipartite quantum system can be quantified, for example, by means of the measure $\mathcal{J}_{S}(\rho)$ proposed by Henderson and Vedral in ref. [30]. Thus, whenever total correlations are measured with mutual information $\mathcal{I}(\rho)$ and classical correlations are measured according to the aforementioned quantity $\mathcal{J}_{S}(\rho)$ (cf. 
Sec. IIB), the measure of quantum correlations that results is the widely known definition of quantum discord (QD) [30, 33, 34], i.e.,

$$
\mathcal{D}(\rho)=\mathcal{I}(\rho)-\mathcal{J}_{S}(\rho) .
$$

As we will see in section $11 \mathrm{~B}$, relation (11) lies at the core of the theoretical framework used in the present paper. However, it should be noticed that Eq. (11) may or may not be satisfied, depending on the approach used to quantify the different type of correlations present in the system under study. For example, when a geometric scheme is used, such as the one introduced in [35], equation (11) is not satisfied. Though, it may be mentioned that a geometric approach to determine correlations should be used with caution since in general it presents some peculiar behaviors, such as those discussed in references [36 38], for example. It can be shown that the essence of QD, as defined by Eq. (44), is to quantify the discrepancy between the quantum versions of two classically equivalent expressions for mutual information [30, 33]. However, although from a conceptual point of view QD is of relevance in assessing possible non-classical resources for information processing, for practical use it presents some drawbacks. For example, at this moment there is no straightforward criterion to verify the presence of nonzero discord in a given arbitrary bipartite quantum state (i.e., an arbitrary bipartite state belonging to the product of two Hilbert spaces of arbitrary dimensions). Besides, as the evaluation of QD involves an optimization procedure, analytical results are only known in some particular cases [34, 39 47]. Furthermore, in general, calculation of quantum discord is NP-complete since the optimization procedure needs to be done by means of a sweep over a complete set of measurements performed over one of the subsystems [48]. Additionally, quantum discord can be created starting from a state with zero discord and then performing Local Operations [49, 50]. It is hard to accept that local operations alone do increase the amount of correlations between two subsystems. Instead, it is expected that correlations contained in the resulting state are lower than or equal to the correlations of the classical state. As this last statement is not reflected in the behaviour of quantum discord, it turns out to be questionable to affirm that (all) discordant states can be thought of as resources useful for quantum information processing tasks. Therefore, a central problem in quantum physics is to be able to characterize and quantify correlations in multipartite states. Further research on correlations is important not only from a practical point of view but also from a conceptual point of view as they 
can provide additional insights about the underlying physics behind correlations present in quantum systems. Detecting the existence of non-classical correlations in a given system is the central goal for a measure of correlations regarding the possibility of classifying a given system as a genuinely quantum one. To this end, it may result of interest to devise proper means to detect and quantify the presence of such correlations. Measures of different type of correlations in quantum systems can be motivated by different notions of classicality or operational means to quantify nonclassicality. Thus, measures of quantum correlations may in general differ, both formally and conceptually. Regarding quantum discord, for example, it can be seen that it does not coincide in general with entanglement or measurement induced disturbance [35, 51, 52]. Moreover, a direct comparison between these two notions may be meaningless [35, 53]. As a result, general approaches and theoretical frameworks seem to be central to the study and understanding of different notions of quantumness.

In previous works, some authors thoroughly discussed a set of properties that bonafide measures of correlations should satisfy [27, 54, 55]. In this work we introduce a scheme to measure correlations in bipartite quantum systems in the following way: Starting from the (plausible) definition of a measure of total correlations $\mathcal{T}(\rho)$ and a measure of classical correlations, we build a measure $\mathcal{Q}(\rho)$ of quantum correlations based on the difference between the two former. Then, based on refs. [54] and [55], we show that the measure $\mathcal{Q}(\rho)$ satisfies a set of desirable properties for a suitable measure of quantum correlations. In this way, our proposal for measures of different type of correlations in a bipartite quantum system turns out to be consistent.

This paper is organized as follows. In Sec. II, we outline the basic theoretical background directly related to our work. In Sec. III, we develop our main results, i.e., we introduce a general approach to define measures of different type of correlations using the concept of distinguishability between quantum states. Finally, some conclusions are drawn in Sec. [V]

\section{THEORETICAL FRAMEWORK}

\section{A. Distance measures in the quantum realm}

From a conceptual point of view, a metric $d\left(. \|\right.$.) on a set $\chi$ is a functional $d: \chi \times \chi \rightarrow \mathbb{R}_{\geq 0}$ such that for every $x, y, z \in \chi$ the following properties are satisfied: 
a) Non-negativity: For any $x, y \in \chi, d(x \| y) \geq 0$

b) Identity of indiscernibles: $d(x \| y)=0$ if and only if $x=y$

c) Symmetry: For any $x, y \in \chi, d(x \| y)=d(y \| x)$

d) Triangle inequality For any $x, y, z \in \chi, d(x \| y) \leq d(x \| z)+d(z \| y)$

In the context of probability theory, a functional $d$ satisfying properties a) and b) is called a divergence. If, in addition, $d$ satisfies property c), then $d$ is called a distance.

In quantum physics, the divergences and distances are defined on the set of quantum states represented by density operators acting on a Hilbert space $\mathcal{H}$. Density operators representing the states of a given system are elements belonging to $\mathcal{B}_{1}^{+}(\mathcal{H})$, i.e., the set of bounded, positive-semidefinite operators on $\mathcal{H}$, with unit trace. In particular, unit vectors in $\mathcal{H}$ correspond to the extremal elements of $\mathcal{B}_{1}^{+}(\mathcal{H})\left(\rho \in \mathcal{B}_{1}^{+}(\mathcal{H})\right.$ is extremal if and only if it is idempotent, i.e., $\rho^{2}=\rho$ ). Any pure state corresponds to a unit vector $|\varphi\rangle \in \mathcal{H}$ and can be represented by a density matrix of the form $\rho=|\varphi\rangle\langle\varphi|$.

One of the most striking features of quantum mechanics is that, in general, two arbitrary quantum states cannot be distinguished with certainty. Only orthogonal states can be discriminated unambiguously. Therefore, in order to determine how close two quantum states are to each other, a variety of distance measures have been developed, as for example, trace distance, Fidelity, Bures distance, Hilbert-Schmidt distance, Hellinger distance and Quantum Jensen-Shannon divergence, just to name a few [56 63].

From physical grounds, the following properties are also usually required for a distance between quantum states to be well-behaved [56]:

e) $d(\cdot \| \cdot)$ must be invariant under unitary transformations, i.e.

$$
d\left(U \rho U^{\dagger} \| U \sigma U^{\dagger}\right)=d(\rho \| \sigma),
$$

being $U$ some unitary operator;

f) $d(\cdot \| \cdot)$ must be non-increasing under the action of a trace-preserving quantum operation,

$$
d(\mathcal{E}(\rho) \| \mathcal{E}(\sigma)) \leq d(\rho \| \sigma),
$$


g) $d(\cdot \| \cdot)$ must be convex in one of its inputs,

$$
d\left(\sum_{i} p_{i} \rho_{i} \| \sigma\right) \leq \sum_{i} p_{i} d\left(\rho_{i} \| \sigma\right)
$$

being $\left\{p_{i}\right\}$ a probability distribution and $\left\{\rho_{i}\right\}$ elements of $\mathcal{B}_{1}^{+}(H)$;

In addition, as we shall discuss in Sec $\underline{\text { IIC }}$, the following property shall be required for well-behaved measures of quantum correlations:

h) $d(\cdot \| \cdot)$ must satisfy

$$
d\left(\sum_{i} p_{i}|i\rangle\left\langle i\left|\otimes \rho_{i} \| \sum_{j} p_{j}\right| j\right\rangle\langle j| \otimes \rho\right)=\sum_{k} p_{k} d\left(\rho_{i}|| \rho\right),
$$

being $\{|i\rangle\}$ an orthonormal basis of $\mathcal{H}_{A},\left\{p_{i}\right\}$ a probability distribution and $\left\{\rho_{i}\right\}$ elements of $\mathcal{B}_{1}^{+}(H)$ such that $\sum_{i} p_{i} \rho_{i}=\rho$.

\section{B. Correlations in quantum systems}

Let us consider a bipartite system $(A+B)$ in an arbitrary quantum state $\rho$ belonging to $\mathcal{B}_{1}^{+}\left(\mathcal{H}_{A B}\right)$, being $\mathcal{H}_{A B}=\mathcal{H}_{A} \otimes \mathcal{H}_{B}$ and $\mathcal{H}_{A}$ and $\mathcal{H}_{B}$ the Hilbert spaces associated with subsystems $A$ and $B$, respectively.

If the system is prepared in a pure state $|\psi\rangle_{A B} \in \mathcal{H}_{A B}$, (i.e., $\rho=\left|\psi_{A B}\right\rangle\left\langle\psi_{A B}\right|$ ) we have essentially two relevant physical scenarios. In the first one, the subsystems are independent (i.e., there are no correlations of any type between both subsystems). In this case, the state can be described as a tensor product, i.e., $|\psi\rangle_{A B}=|\phi\rangle_{A} \otimes|\chi\rangle_{B}$. In the second scenario, the global state cannot be written as a tensor product, i.e., $|\psi\rangle_{A B} \neq|\phi\rangle_{A} \otimes|\chi\rangle_{B}$. In this case, the state is said to be entangled. Entanglement is the unique form of quantum correlation that might take place in the case of bipartite pure states. Moreover, entanglement, non locality, and QCs are synonymous in this particular case.

In contrast, for a system prepared in a mixed state described by a density matrix $\rho$, other type of correlations can be involved. In this case we are in the presence of a subtler and richer situation than the case of pure states. Indeed, unentangled mixed states can exhibit correlations of a quantum nature [15, 22]. 
The set of separable (unentangled) states $\mathcal{S}_{A, B}$ is composed by density operators $\rho \in$ $\mathcal{B}_{1}^{+}\left(\mathcal{H}_{A B}\right)$ such that

$$
\mathcal{S}_{A, B} \doteq\left\{\rho \in \mathcal{B}_{1}^{+}\left(\mathcal{H}_{A B}\right) / \rho=\sum_{i} p_{i} \rho_{A}^{i} \otimes \rho_{B}^{i}\right\}
$$

where $\rho_{A(B)}^{i}$ is associated with the quantum state of subsystem $A(B)$ and $\left\{p_{i}\right\}$ are nonnegative numbers such that $\sum_{i} p_{i}=1$. The set of product states (which is included in the set of separable states) is defined as follows

$$
\mathcal{P}_{A, B} \doteq\left\{\rho \in \mathcal{B}_{1}^{+}\left(\mathcal{H}_{A B}\right) / \rho=\rho_{A} \otimes \rho_{B}\right\} .
$$

A state $\rho$ belonging to the set $\mathcal{P}_{A, B}$ is called uncorrelated in the sense that it does not contain correlations of any type whatsoever. Then, a given quantum state $\rho$ can be a separable or a product state if it can be reduced to the form established in equations (9) or (10), respectively.

Any state $\rho$ that can not be written in the form given by Eq. (9) is referred to as nonseparable. It is important to realize that only non-separable states have quantum correlations analogous to that of an entangled pure state. However, non-classical correlations may be present in some subset of separable states. Regarding a bipartite system where subsystems $A$ and $B$ are classically correlated, it can be shown that the global state $\rho$ belong to the following set:

$$
\mathcal{C}_{A, B} \doteq\left\{\rho \in \mathcal{B}_{1}^{+}\left(\mathcal{H}_{A B}\right) / \rho=\sum_{i, j} p_{i, j}|i\rangle\left\langle\left. i\right|_{A} \otimes \mid j\right\rangle\left\langle\left. j\right|_{B}\right\}\right.
$$

being $\left\{|i\rangle_{A}\right\}$ and $\left\{|j\rangle_{B}\right\}$ orthonormal bases of the Hilbert spaces $\mathcal{H}_{A}$ and $\mathcal{H}_{B}$, respectively, and $\left\{p_{i j}\right\}$ a joint probability distribution. Furthermore, the set of (asymmetric) classicalquantum states is defined as follows,

$$
\mathcal{C}_{A}:=\left\{\rho \in \mathcal{B}_{1}^{+}\left(\mathcal{H}_{A B}\right) / \rho=\sum_{i} p_{i}|i\rangle\left\langle\left. i\right|_{A} \otimes \rho_{B}^{i}\right\}\right.
$$

where $\left\{p_{i}\right\}$ is a probability distribution and $\left\{\rho_{B}^{i}\right\}$ any set of quantum states of the subsystem $B$. The set $\mathcal{C}_{B}$ of quantum-classical states can be analogously defined.

Total correlations given by Eq. (22) can be recast in terms of von Neumann relative entropy as follows:

$$
I(\rho)=S\left(\rho \| \rho_{A} \otimes \rho_{B}\right),
$$


where relative entropy is given by

$$
S(\rho \| \sigma)=\operatorname{Tr}[\rho(\ln \rho-\ln \sigma)] .
$$

Thus, Eq.(13) can be interpreted as the distance between the state $\rho$ and the uncorrelated state $\rho_{A} \otimes \rho_{B}$. In this case, the distance is quantified by means of quantum relative entropy.

According to ref. [30], the measure of classical correlations $\mathcal{J}_{S}(\rho)$ involved in Eq. (44) is defined as

$$
\mathcal{J}_{S}(\rho)=S\left(\rho_{B}\right)-\min _{M_{j}} \sum_{j} p_{j}^{\prime} S\left(\rho_{B \mid j}^{\mathcal{M}}\right),
$$

being $\mathcal{M}=\left\{M_{j}\right\}_{j=1}^{m}(m \in \mathbb{N})$, a von Neumann measurement on subsystem $A$ (i.e., a family of orthonormal projectors on $\mathcal{H}_{A}$ ) and being

$$
\begin{array}{r}
\rho_{B \mid j}^{\mathcal{M}}=\operatorname{Tr}_{A}\left[M_{j} \otimes \mathbb{I} \rho\right] / p_{j}^{\prime} ; \\
p_{j}^{\prime}=\operatorname{Tr}\left[M_{j} \otimes \mathbb{I} \rho\right],
\end{array}
$$

the states of the subsystem $B$ and the conditional probabilities of getting the state $\rho_{B \mid j}^{\mathcal{M}}$ after the measurement $\mathcal{M}$, respectively. From its definition (cf. Eq. (15)), it can be seen that the measure $\mathcal{J}_{S}(\rho)$ it is not symmetric under the exchange of subsystems $A$ and $B$. Therefore, in some sense there exists a directionality over $\mathcal{J}_{S}(\rho)$ and, as a consequence over the quantity $\mathcal{D}(\rho)$. States given by Eq. (16) are commonly referred to as conditional states.

From Eq. (1) we can define a directional measure of quantum correlations in the form

$$
\mathcal{Q}(\rho) \doteq \mathcal{T}(\rho)-\mathcal{J}(\rho)
$$

From last equation, it can be easily seen that QD is a particular case of a measure of quantum correlations when we choose $\mathcal{T}(\rho)=\mathcal{I}(\rho)$ (cf. eqs. (2) ) and (13) $)$ and $\mathcal{J}(\rho)=\mathcal{J}_{S}(\rho)$ (cf. Eq. (15)). Bearing in mind (18) we will introduce in Sec $\llbracket$ III a general approach to define measures of different types of correlations based upon the concept of distinguishability between states in the quantum realm.

We would like to emphasize that $\mathcal{J}(\rho)$ is not a symmetric quantity under the exchange of $A$ for $B$. Therefore, $\mathcal{J}(\rho)$ is in fact a measure of classical-quantum correlations.

\section{Properties of correlation measures}

The set of criteria to be satisfied for valid quantifiers of correlations in quantum systems is still a subject of active research. However, following refs. [54] and [55] we shall require 
the following set of necessary properties for any well-behaved measure of correlations:

1. Product states have no correlations, then: $\mathcal{Q}\left(\rho_{A} \otimes \rho_{B}\right)=\mathcal{T}\left(\rho_{A} \otimes \rho_{B}\right)=\mathcal{J}\left(\rho_{A} \otimes \rho_{B}\right)=0$;

2. All correlation measures should be invariant under local unitary operations;

3. All correlation measures should be non-negative, i.e., $\mathcal{Q}(\rho) \geq 0, \mathcal{T}(\rho) \geq 0$ and $\mathcal{J}(\rho) \geq$ 0

4. Total correlations measures should be non-increasing under local operations;

5. Classical states have no quantum correlations.

According to ref. [54], any measure which does not meet these necessary criteria is not a valid measure of correlations.

\section{GENERALIZED CORRELATION MEASURES IN BIPARTITE QUANTUM SYSTEMS}

In this section we propose measures of total, classical and quantum correlations. Also, taking into account the criteria previously introduced (cf. Sec【I), we analyze the properties to be satisfied by a distance in order to build proper correlation measures.

\section{A. Generalized measures of total correlations}

Equation (13) provides the fundamental basis to define measures of total correlations by means of a distance between quantum states. Thus, given an arbitrary distance $d(\cdot \| \cdot)$ we propose a measure of total correlations as follows:

$$
\mathcal{T}_{d}(\rho)=d\left(\rho \| \rho_{A} \otimes \rho_{B}\right) .
$$

The relation (19) provides a whole new family of quantifiers which, in principle, might be used as tools for analyzing different possible scenarios of physical relevance.

Now, in order to prove that the measure $\mathcal{T}_{d}(\rho)$ quantifies total correlations, we shall study how the properties imposed on $d(\cdot \| \cdot)$ are related to the properties to be fulfilled by $\mathcal{T}_{d}(\rho)$. Thus, we establish the following propositions: 
Proposition I If $d(\cdot \| \cdot)$ satisfies a), b) and e) (cf. Sec [IA) then $\mathcal{T}_{d}$ fulfills the conditions [1] (cf. Sec $\underline{\text { IIC) }}$.

Proposition II If, in addition, $d(\cdot \| \cdot)$ also satisfies the $\mathrm{f}$ ) (cf. Sec $\underline{\text { IIA }}$ ) then $\mathcal{T}_{d}$ fulfills the property 4 (cf. Sec.IC).

The proofs of both propositions are straightforward.

\section{B. Generalized measures of classical-quantum correlations}

In this section our aim is to introduce classical-quantum correlations measures for a bipartite arbitrary state. The basic idea is to apply a measurement $\mathcal{M}$ on subsystem $A$ and see afterwards how this action conditions the state of subsystem $B$. Then, we shall quantify how different is the state $\rho_{B}$ from the states $\rho_{B \mid j}^{\mathcal{M}}$ arising after performing the local measurement on $A$, by means of the distance $d\left(\rho_{B \mid j}^{\mathcal{M}} \| \rho_{B}\right)$. Thus, a measure $\mathcal{J}_{d}(\rho)$ of correlations between the two subsystems shall be the maximum of the weighted average of the above $m \in \mathbb{N}$ quantities $d\left(\rho_{B \mid j}^{\mathcal{M}} \| \rho_{B}\right)$, i.e.,

$$
\mathcal{J}_{d}(\rho):=\max _{\{\mathcal{M}\}} \mathcal{J}_{d}^{\mathcal{M}}(\rho)
$$

being $\mathcal{J}_{d}^{\mathcal{M}}(\rho)$ defined as follows

$$
\mathcal{J}_{d}^{\mathcal{M}}(\rho):=\sum_{j=1}^{m} p_{j}^{\prime} d\left(\rho_{B \mid j}^{\mathcal{M}} \| \rho_{B}\right) .
$$

Looking at equations (20) and (21), we notice that expression (15) is recovered whenever the distance $d(\cdot \| \cdot)$ is replaced with the relative entropy.

Now, we shall study the necessary properties to be fulfilled by $d(\cdot \| \cdot)$ in order for $\mathcal{J}_{d}$ to meet the criteria for classical-quantum correlation measures. With this aim, we establish the following proposition.

Proposition III If $d(\cdot \| \cdot)$ satisfies properties a), b) and e) (cf. Sec. IIA), then $\mathcal{J}_{d}$ fulfills the conditions 1 , 3 (cf. Sec. 【C).

Proof: Let us suppose that the system is in a product state $\sigma=\sigma_{A} \otimes \sigma_{B}$. In this case, it can be seen that $\sigma_{B \mid j}^{\mathcal{M}}=\sigma_{B}$ for any measurement $\mathcal{M}$. Thus, if the distance $d$ satisfy $[$ ) (cf. 
Sec. (IA) it follows that $d\left(\sigma_{B \mid j}^{\mathcal{M}} \| \sigma_{B}\right)=0$. Therefore, we have shown that $\mathcal{J}_{d}(\sigma)=0$, and the requirement 1 is fulfilled.

Next, we shall prove that requirement 2 is satisfied by $\mathcal{J}_{d}(\rho)$ whenever the distance $d$ satisfies property e), i.e., it remains invariant under local unitary transformations.

Indeed, let us consider $\sigma=U \rho U^{\dagger}$ with $U=U_{A} \otimes U_{B}$. Then, $\sigma_{B \mid j}^{\mathcal{M}}=U_{B} \rho_{B \mid j}^{\mathcal{M}^{\prime}} U_{B}^{\dagger}$ and $\sigma_{B}=U_{B} \rho_{B} U_{B}^{\dagger}$, being $\mathcal{M}^{\prime}=\left\{U_{A} M_{j} U_{A}^{\dagger}\right\}$. Thus, it follows that

$$
d\left(\sigma_{B \mid j}^{\mathcal{M}} \| \sigma_{B}\right)=d\left(U_{B} \rho_{B \mid j}^{\mathcal{M}^{\prime}} U_{B}^{\dagger} \| U_{B} \rho_{B} U_{B}^{\dagger}\right)=d\left(\rho_{B \mid j}^{\mathcal{M}^{\prime}} \| \rho_{B}\right)
$$

Furthermore, it can be seen that the probability distribution $p_{j}^{\prime}$ for the state $\sigma$ is $p_{j}^{\prime}=$ $\operatorname{Tr}\left[M_{j} \otimes \nVdash \sigma\right]=\operatorname{Tr}\left[M_{j}^{\prime} \otimes \nVdash \rho\right]$. Therefore,

$$
\mathcal{J}_{d}^{\mathcal{M}}(\sigma)=\mathcal{J}_{d}^{\mathcal{M}^{\prime}}(\rho)
$$

Due to $\mathcal{M}^{\prime}$ is composed by each one of the elements of $M$ transformed upon the action of the unitary operation $U_{A}$ on party $A$, we have:

$$
\mathcal{J}_{d}(\sigma)=\max _{\{\mathcal{M}\}} \mathcal{J}_{d}^{\mathcal{M}}(\sigma)=\max _{\{\mathcal{M}\}} \mathcal{J}_{d}^{\mathcal{M}^{\prime}}(\rho)=\max _{\{\mathcal{M}\}} \mathcal{J}_{d}^{\mathcal{M}}(\rho)=\mathcal{J}_{d}(\rho)
$$

Thus, we have shown that property 2 is satisfied by $\mathcal{J}_{d}(\rho)$.

Finally, the positivity of $\mathcal{J}_{d}(\rho)$ (cf. Sec $\llbracket$ IIC, property 3 follows from equations (20) and (21) and the positivity of $d$ (cf. a), Sec $\underline{\text { IA }}$ )

In order for $\mathcal{J}_{d}$ to be a measure of classical-quantum correlations, let us consider the following classical-quantum state $\rho_{c}=\sum_{i} p_{i}|i\rangle\left\langle\left. i\right|_{A} \otimes \rho_{B}^{i}\right.$. Then, the following condition needs to be satisfied,

$$
\mathcal{J}_{d}\left(\rho_{c}\right)=\sum_{i} p_{i} d\left(\rho_{B}^{i} \| \rho_{B}\right)
$$

This last equation follows from the fact that for the state $\rho_{c}$ the best measurement we can perform on the subsystem $A$ to obtain information about the subsystem $B$ is a projective measurement on the states $\{|i\rangle\}$ [30], i.e., $\mathcal{M}=\{|i\rangle\langle i|\}$ maximizes (21), therefore Eq. (20) should match Eq. (25) for $\rho_{c}$.

Next, we shall study the properties to be satisfied by $d(\cdot \| \cdot)$ in order for $\mathcal{J}_{d}$ to fulfill equation (25). Thus, we establish the following proposition: 
Proposition IV If $d(\cdot \| \cdot)$ is convex in one of its inputs, (cf. property g), Sec. IIA), then $\mathcal{J}_{d}\left(\rho_{c}\right)$ shall satisfy Eq. (25).

Proof: The conditional states $\rho_{B \mid j}^{\mathcal{M}}$ can be written as

$$
\rho_{B \mid j}^{\mathcal{M}}=\sum_{i} \frac{p_{i} q_{i j}}{p_{j}^{\prime}} \rho_{B}^{i}
$$

being $p_{j}^{\prime}=\sum_{i} p_{i} q_{i j}$ and $q_{i j}=\operatorname{Tr}\left[M_{j}|i\rangle\left\langle\left. i\right|_{A}\right]\right.$. Therefore, we have

$$
\mathcal{J}_{d}^{\mathcal{M}}\left(\rho_{c}\right)=\sum_{i} p_{j}^{\prime} d\left(\sum_{i} \frac{p_{i} q_{i j}}{p_{j}^{\prime}} \rho_{B}^{i} \| \rho_{B}\right) .
$$

As the distance $d(\cdot \| \cdot)$ fulfills property $\mathrm{g})$, the following inequality holds,

$$
d\left(\sum_{i} \frac{p_{i} q_{i j}}{p_{j}^{\prime}} \rho_{B}^{i} \| \rho_{B}\right) \leq \sum_{i} \frac{p_{i} q_{i j}}{p_{j}^{\prime}} d\left(\rho_{B}^{i} \| \rho_{B}\right)
$$

Using that $\sum_{j} M_{j}=\nVdash$ and $\operatorname{Tr}\left[|i\rangle\left\langle\left. i\right|_{A}\right]=1\right.$ it follows that $\sum_{j} q_{i j}=1$. Thus, by multiplying this last inequality with $p_{j}^{\prime}$ and performing the sum over $j$, we obtain

$$
\mathcal{J}_{d}^{\mathcal{M}}\left(\rho_{c}\right) \leq \sum_{i} p_{i} d\left(\rho_{B}^{i}|| \rho_{B}\right)
$$

It should be noticed that we have not imposed the condition of symmetry on $d(\cdot \| \cdot)$. Therefore, according to the last proposition, the conditional states $\rho_{B \mid j}^{\mathcal{M}}$ must enter in the argument for which the distance is convex.

Next, in order to verify that $\mathcal{J}_{d}$ is a measure of classical-quantum correlations, we will consider an arbitrary state $\rho$ and we will perform a von Neumann measurement $\mathcal{M}=$ $\left\{M_{j}\right\}_{j=1}^{m}$ on the subsystem $A$. The state of the system after the measurement (with unknown result, i.e., the observers do not have access to the measurement outcome) is given by

$$
\rho^{\mathcal{M}}=\sum_{j} M_{j} \otimes \nVdash \rho M_{j} \otimes \nVdash
$$

where $\rho^{\mathcal{M}} \in \mathcal{C}_{A}$ is a classical-quantum state. This state can also be written as,

$$
\rho^{\mathcal{M}}=\sum_{j} p_{j}^{\prime} M_{j} \otimes \rho_{B \mid j}^{\mathcal{M}}
$$


where $\rho_{B \mid j}^{\mathcal{M}}$ and $p_{j}^{\prime}$ are defined in equations (16) and (17), respectively. Then, using Eq. (25) and the convexity of $d(\cdot \| \cdot)$ with respect to its first input, we have

$$
\mathcal{J}_{d}\left(\rho^{\mathcal{M}}\right)=\sum_{j=1} p_{j}^{\prime} d\left(\rho_{B \mid j}^{\mathcal{M}} \| \rho_{B}\right)=\mathcal{J}_{d}^{\mathcal{M}}(\rho)
$$

The state $\rho^{\mathcal{M}}$ represents a classical-quantum correlated state, in the sense that the correlations are between a classical subsystem $A$ and a quantum subsystem $B$. Since $\rho$ is an arbitrary state, equation (32) allows us to conclude that $\mathcal{J}_{d}$ genuinely quantifies classicalquantum correlations.

\section{Generalized measures of quantum correlations}

Having defined generalized measures of total and classical-quantum correlations and keeping in mind Eq.(18), we propose a measure of quantum correlations as follows,

$$
\mathcal{Q}_{d}(\rho):=\mathcal{T}_{d}(\rho)-\mathcal{J}_{d}(\rho)
$$

From previous sections we know that if $d(\cdot \| \cdot)$ satisfies conditions a), b) and e) IIA) then $\mathcal{T}_{d}$ and $\mathcal{J}_{d}$ satisfy the necessary conditions 1 - 4 for correlation measures (cf. Sec. IC). In order for $\mathcal{Q}_{d}$ to fulfill the necessary criteria (cf. Sec. IC), we need to impose an extra condition on $d(\cdot \| \cdot)$. Thus, we introduce the following proposition:

Proposition V If $d(\cdot \| \cdot)$ has the properties a), b) and e) h) (cf. Sec. II A) then $\mathcal{Q}_{d}$ meets $1+3$ and 5 (cf. Sec. IC).

Proof: The proof of this last proposition relies upon the consistency of the scheme established by the measures $\mathcal{T}_{d}, \mathcal{J}_{d}$, and $\mathcal{Q}_{d}$. Thus, we shall prove next that $\mathcal{Q}_{d}$ is positivesemidefinite.

For the classical-quantum state $\rho^{\mathcal{M}}$ obtained after measurement, given by Eq. (30), we can write the following inequality,

$$
\mathcal{T}_{d}(\rho) \geq \mathcal{T}_{d}\left(\rho^{\mathcal{M}}\right)
$$

As $\rho^{\mathcal{M}} \in \mathcal{C}_{A}$, according to the property $\left.\mathrm{h}\right)$ of $d(\cdot \| \cdot)$ it follows that

$$
\mathcal{T}_{d}\left(\rho^{\mathcal{M}}\right)=\mathcal{J}_{d}\left(\rho^{\mathcal{M}}\right)
$$


or equivalently according with Eq. (32)

$$
\mathcal{T}_{d}\left(\rho^{\mathcal{M}}\right)=\mathcal{J}_{d}^{\mathcal{M}}(\rho)
$$

Then, using Eq. (34) we can write

$$
\mathcal{T}_{d}(\rho) \geq \mathcal{J}_{d}^{\mathcal{M}}(\rho)
$$

for any von Neumann measurement $\mathcal{M}$, in particular for the one that maximizes the classicalquantum correlations. Thus, the condition

$$
\mathcal{T}_{d}(\rho) \geq \mathcal{J}_{d}(\rho)
$$

is satisfied and the positivity of $\mathcal{Q}_{d}$ has been demonstrated.

The proof that $\mathcal{Q}_{d}$ satisfies requirement 5 follows from property $\mathrm{h}$ ) and Eq. (32). In addition, due to $\mathcal{T}_{d}$ and $\mathcal{J}_{d}$ are invariant under local unitary transformations and both yield zero when evaluated in product states, it follows that $\mathcal{Q}_{d}$ also satisfy properties 1 and 2 .

It should be noticed that the measure of quantum correlations $\mathcal{Q}_{d}$ reduces to quantum discord in the case $d(\cdot \| \cdot)=S(\cdot \| \cdot)$, being $S$ the quantum relative entropy.

\section{Examples}

Previously, we obtained a set of requirements for a quantum distance in order to build well-behaved quantifiers of correlations between subsystems (cf. Sec.III). In the case of generalized measures of total and classical-quantum correlations, on the one hand, our results show that distance $d$ should satisfy properties a), b) and e). On the other hand, in the case of quantum correlations measures, distance $d$ should also satisfy property h) (cf. Sec. ЩA). Now, besides the well-known case of relative entropy, we shall consider the following particular examples: the trace distance, the squared Bures distance, the quantum Jensen-Shannon divergence (QJSD) and the squared Hellinger distance. All these distances fulfill the conditions a), b) and e) [56, 63, 64]. The only requirement not considered in literature is h). Therefore we will prove this last property for the cases of trace distance and Quantum Jensen Shannon divergence. The remaining cases can be easily proved using similar arguments to those of trace distance. 


\section{Trace distance:}

If $\rho_{1}$ and $\rho_{2}$ are two density matrices, then trace distance is defined as follows: [56]:

$$
d_{T r}\left(\rho_{1} \| \rho_{2}\right)=\frac{1}{2} \operatorname{Tr}\left[\sqrt{\left(\rho_{1}-\rho_{2}\right)^{2}}\right] .
$$

Let us choose $\rho_{1}=\sum_{i} p_{i} E_{i} \otimes \rho_{i}$ and $\rho_{2}=\sum_{j} p_{j} E_{j} \otimes \rho$, being $E_{j}=|j\rangle\langle j|$ and $\{|j\rangle\}$ an orthonormal basis of the subsystem $A$. Then,

$$
\left(\rho_{1}-\rho_{2}\right)^{2}=\sum_{i} p_{i}^{2} E_{i} \otimes\left(\rho_{i}-\rho\right)^{2} .
$$

As $\left(\sum_{i} p_{i} E_{i} \otimes \sqrt{\left(\rho_{i}-\rho\right)^{2}}\right)^{2}=\sum_{i} p_{i}^{2} E_{i} \otimes\left(\rho_{i}-\rho\right)^{2}$ then

$$
\sqrt{\left(\rho_{1}-\rho_{2}\right)^{2}}=\sum_{i} p_{i} E_{i} \otimes \sqrt{\left(\rho_{i}-\rho\right)^{2}} .
$$

Consequently,

$$
d_{T r}\left(\rho_{1} \| \rho_{2}\right)=\frac{1}{2} \sum_{i} p_{i} \operatorname{Tr}\left[\sqrt{\left(\rho_{i}-\rho\right)^{2}}\right]=\sum_{i} p_{i} d_{T r}\left(\rho_{i} \| \rho\right) .
$$

\section{Quantum Jensen Shannon divergence:}

The QJSD is defined as follow

$$
d_{j s}\left(\rho_{1} \| \rho_{2}\right)=S\left(\frac{\rho_{1}+\rho_{2}}{2}\right)-\frac{1}{2} S\left(\rho_{1}\right)-\frac{1}{2} S\left(\rho_{2}\right)
$$

being $S(\cdot)$ the von Neumann entropy which yields the following result when evaluated in classical-quantum states:

$$
S\left(\rho_{1}\right)=-\operatorname{Tr}\left[\rho_{1} \log \rho_{1}\right]=H(p)+\sum_{i} p_{i} S\left(\rho_{i}\right)
$$

In last equation, $H(p)=-\sum_{i} p_{i} \log p_{i}$ represents the Shannon entropy. Thus,

$$
\begin{aligned}
& S\left(\frac{\rho_{1}+\rho_{2}}{2}\right)=H(p)+\sum_{i} p_{i} S\left(\frac{\rho_{i}+\rho}{2}\right) \\
& S\left(\rho_{1}\right)=H(p)+\sum_{i} p_{i} S\left(\rho_{i}\right) \\
& S\left(\rho_{2}\right)=H(p)+\sum_{i} p_{i} S(\rho) .
\end{aligned}
$$

It immediately follows that

$$
d_{j s}\left(\rho_{1} \| \rho_{2}\right)=\sum_{i} p_{i}\left[S\left(\frac{\rho_{i}+\rho}{2}\right)-\frac{1}{2} S\left(\rho_{i}\right)-\frac{1}{2} S(\rho)\right]=\sum_{i} p_{i} d_{j s}\left(\rho_{i} \| \rho\right)
$$




\section{Additional conditions for quantum correlations measures}

In reference [55] Adesso et al. proposed three additional conditions that should be satisfied for well-behaved measures of quantum correlations. Two of them are related to the behavior of the measure under local quantum operations. The other one states that for a pure state the measure should reduce to an entanglement quantifier.

According to ref. [55], it should be impossible to create quantum correlations by performing operations only on party B of the bipartite system. In this context, we were able to prove the following proposition:

Proposition VI If $d(\cdot \| \cdot)$ satisfy the conditions b) h) (cf. Sec. IIA) in addition to the following two properties:

- Restricted Additivity, $d\left(\rho_{1} \otimes \sigma \| \rho_{2} \otimes \sigma\right)=d\left(\rho_{1} \| \rho_{2}\right)$ where $\rho_{1}, \rho_{2}$ and $\sigma$ belong to $\mathcal{B}_{1}^{+}(\mathcal{H})$.

- Given $\sigma_{A B E} \in \mathcal{B}_{1}^{+}\left(\mathcal{H}_{A B E}\right), \mathcal{H}=\mathcal{H}_{\mathcal{A}} \otimes \mathcal{H}_{\mathcal{B}} \otimes \mathcal{H}_{E}$, the quantity

$$
d\left(\sigma_{A B E} \| \sigma_{A E} \otimes \sigma_{B}\right)-d\left(\sigma_{A B} \| \sigma_{A} \otimes \sigma_{B}\right)
$$

is invariant under the exchange of the subsystems $B$ and $E$. Here, the matrices $\sigma$ in the arguments of $d(\cdot \| \cdot)$ are the reduced matrices of $\sigma_{A B E}$, for example: $\sigma_{A E}=\operatorname{Tr}_{B}\left[\sigma_{A B E}\right]$.

then $\mathcal{Q}_{d}(\rho)$ is monotonic non-increasing under local operations on subsystem $B$ (cf. property 4, Sec. 【IC).

Proof: In reference [? ] it is demonstrated that quantum discord satisfies property 4, The proof makes use of the properties of von Neumann entropy. Proposition (VI) can be proved following the same lines of reasoning as in ref. [? $]$

If some of the assumptions in proposition (VI) are relaxed, it may happen that the monotonically non-increasing behavior of $\mathcal{Q}_{d}(\rho)$ remain valid. In such a case, a particular analysis should be necessary for the particular choice of the distance $d$.

Finally, it is important to study the conditions that the distance $d$ should satisfy so that $\mathcal{Q}_{d}$ is reduced to a physically well-behaved entanglement measure in the case of pure states. This is not an easy task and it will be the subject of future research. 


\section{CONCLUDING REMARKS}

In this work, we proposed a general approach to the problem of quantifying different types of correlations in bipartite quantum systems through the use of distances between quantum states and processes. Under the assumption that total correlations contained in a bipartite state can be assessed as a sum of classical and quantum contributions, we analyzed the properties that distances must fulfill in order to derive well-behaved quantifiers of correlations. On the one hand, we demonstrated that distances satisfying basic properties, namely non-negativity, identity of indiscernibles, and invariance under unitary transformations, in addition to the requirement of being non-increasing under the action of trace-preserving quantum operations (cf. Sec. IIA properties a), b), e), and f)], can be used to build physically well-behaved measures of total correlations. On the other hand, in order to obtain well-behaved measures of classical-quantum correlations, distances must verify, in addition to the basic properties, the requirement of being convex in one of its inputs (cf. Sec. IIA, property g) . Furthermore, in order to obtain well-behaved quantum correlation measures, we showed that distances should also satisfy an additional not too restrictive requirement (cf. Sec. IIA, property h). We also showed that the proposed measures of quantum correlations are monotonic non-increasing under local operation on subsystem $B$ whenever the distance measure satisfies the restricted additivity and the relation given by Eq.(49).

Finally, besides the well-known case of relative entropy, we introduced some additional examples of distance measures, i.e., the trace distance, the squared Bures distance, the quantum Jensen-Shannon divergence (QJSD) and the squared Hellinger distance, which can be used to build physically well-behaved quantifiers of correlations. As a result, we showed that it is possible to build quantifiers of different types of correlations within a consistent framework. These quantifiers might be used as additional tools for analyzing different possible scenarios of physical relevance. 


\section{Acknowledgments}

D.B., A.P.M., P.W.L. and T.M.O acknowledge the Argentinian agency SeCyT-UNC and CONICET for financial support. D. B. has a fellowship from CONICET.

[1] Vedral V.: Quantum entanglement. Nat. Phys. 10, 256 (2014)

[2] Yu, T., Eberly, J.H.: The End of an Entanglement. Science 316, 555 (2007)

[3] Yu, T., Eberly, J.H.: Sudden Death of Entanglement. Science 323, 598 (2009)

[4] Plenio, M., S. Virmani: An introduction to entanglement measures. Quantum Inf. Comput. 7, $1(2007)$

[5] Brunner, N., Calvacanti D., Pironio, F., Scarani, V., Wehner S.: Bell nonlocality. Rev. Mod. Phys. 86, 419 (2014)

[6] Orieaux, A., D’Arrigo, A., Ferranti, G., Lo Franco, R., Benenti, G., Paladino, E., Falci, G., Sciarrino, F., Mataloni, P.: Experimental on-demand recovery of entanglement by local operations within non-Markovian dynamics. Scientific Reports 5, 8575 (2015)

[7] Man, Z-X., Xia, Y-J., Lo Franco, R.: Cavity-based architecture to preserve quantum coherence and entanglement. Sci. Rep. 5, 13843 (2015)

[8] Dijkstra, A. G., Tanimura, Y.: Non-Markovian Entanglement Dynamics in the Presence of System-Bath Coherence. Phys. Rev. Lett. 104, 250401 (2010)

[9] Aolina, L. de Melo, F. Davidovich, L.: Open-system dynamics of entanglement: a key issues review. Rep. Prog. Phys. 78, 042001 (2015).

[10] D’Arrigo, A., Lo Franco, R., Benenti, G., Paladino, E., Falci, G.: Hidden entanglement in the presence of random telegraph dephasing noise. Phys. Scr. T153, 014014 (2013)

[11] Xu, J-S., Sun, K., Li, C-F., Xu, X-Y., Guo, G-C., Andersson, E., Lo Franco, R., Compagno, G. : Experimental recovery of quantum correlations in absence of system-environment backaction. Nat. Commun. 4, 2851 (2013).

[12] D'Arrigo, A., Benenti, G., Lo Franco, R., Falci, G.: Hidden entanglement, system-environment information flow and non-Markovianity. Int. J. Quantum Inform. 12, 1461005 (2014)

[13] Lo Franco, R.: Nonlocality threshold for entanglement under general dephasing evolutions: a case study. Quantum Inform. Process. 15, 2393 (2016) 
[14] Rab, A. S., Polino, E., Man, Z-X., An, N. B., Xia, Y-J., Spagnolo, N., Lo Franco, R., Sciarrino, F.: Entanglement of photons in their dual wave-particle nature. Nat. Commun. 8, 915 (2017).

[15] Knill, E., Laflamme, R.: Power of One Bit of Quantum Information. Phys. Rev. Lett. 81, $5672(1998)$

[16] Laflamme, R., Cory, D. G., Negrevergne, C., Viola, L.: NMR quantum information processing and entanglement. Quantum. Inf. and Comp. 2, 166 (2002).

[17] Braunstein, S. L., Caves, C. M., Jozsa, R., Linden, N., Popescu, S., Schack R.: Separability of very noisy mixed states and implications for NMR Quantum computing. Phys. Rev. Lett. 83, 1054 (1999).

[18] Meyer, D., A.: Sophisticated Quantum Search Without Entanglement. Phys. Rev. Lett. 85, $2014(2000)$

[19] Datta, A., Flammia, S., T., Caves, C., M.: Entanglement and the power of one qubit. Phys. Rev. A 72, 042316 (2005)

[20] Datta A., Vidal, G.: Role of entanglement and correlations in mixed-state quantum computation. Phys. Rev. A 75, 042310 (2007)

[21] Datta, A., Shaji, A., Caves, C. M.: Quantum Discord and the Power of One Qubit. Phys. Rev. Lett. 100, 050502 (2008)

[22] Lanyon, B., P., Barbieri, M., Almeida M., P., White, A., G.: Experimental Quantum Computing without Entanglement. Phys. Rev. Lett. 101, 200501 (2008)

[23] Silva, I. A., Souza, A. M., Bromley, T. R., Cianciaruso, M., Marx, R., Sarthour, R., S., Oliveira, I. S., Lo Franco, R., Glaser, S., J., deAzevedo, E., R., Soares-Pinto, D. O., Adesso, G,: Observation of Time-Invariant Coherence in a Nuclear Magnetic Resonance Quantum Simulator. Phys. Rev. Lett. 117, 160402 (2016)

[24] Aaronson, B., Lo Franco, R., \& Adesso G.,: Comparative investigation of the freezing phenomena for quantum correlations under nondissipative decoherence. Phys. Rev. A 88, 012120 (2013)

[25] Aaronson, B., Lo Franco, R., Compagno, G., Adesso, G.: Hierarchy and dynamics of trace distance correlations. New J. Phys. 15093022 (2013)

[26] Haikka, P., Johnson, T. H., Maniscalco, S.: Non-Markovianity of local dephasing channels and time-invariant discord. Phys. Rev. A 87 010103(R) (2013)

[27] Cianciaruso, M., Bromley, T. R., Roga, W., Lo Franco, R., Adesso, G.: Universal freezing of 
quantum correlations within the geometric approach. Sci. Rep. 510177 (2015)

[28] Bennett, C.H., DiVincenzo, D., P., Fuchs, C.A., Mor, T., Rains, E., Shor, P.W., Smolin, J.A., Wootters, W., K.: Mixed State Entanglement and Quantum Error Correction. Phys. Rev. A 591070 (1999)

[29] Groisman, B., Popescu, S., Winter, A.: Quantum, classical, and total amount of correlations in a quantum state. Phys. Rev. A 72, 032317 (2005)

[30] Henderson, L., Vedral, V.: Classical, quantum and total correlations. J. Phys. A 34, 6899 (2001)

[31] Oppenheim, J., Horodecki, M., Horodecki, P., Horodecki, R.: Thermodynamical Approach to Quantifying Quantum Correlations. Phys. Rev. Lett. 89, 180402 (2002)

[32] Yang, D., Horodecki, M., Wang, Z.D.: An Additive and Operational Entanglement Measure: Conditional Entanglement of Mutual Information. Phys. Rev. Lett. 101, 140501 (2008)

[33] Ollivier, H., Zurek, W.H.: Quantum Discord: A Measure of the Quantumness of Correlations. Phys. Rev. Lett 88, 017901 (2001)

[34] Luo S.: Quantum discord for two-qubit systems. Phys. Rev. A 77, 042303 (2008)

[35] Modi, K., Paterek, T., Son, W., Vedral, V., Williamson M.: Unified View of Quantum and Classical Correlations. Phys. Rev. Lett. 104, 080501 (2010)

[36] Piani, M.: Problem with geometric discord. Phys. Rev. A 86, 034101 (2012)

[37] Tufarelli, T., MacLean, T. M., Girolami, D., Vasile, R., Adesso, G.: The geometric approach to quantum correlations: computability versus reliability. J. Phys. A: Math. Theor. 46, 275308 (2013)

[38] Paula, F. M., Saguia A., De Oliveira, T. R., Sarandy, M. S.: Overcoming ambiguities in classical and quantum correlation measures. EPL 108, 10003 (2014)

[39] Lang, M., D., Caves, C., M.: Quantum Discord and the Geometry of Bell-Diagonal States. Phys. Rev. Lett. 105, 150501 (2010)

[40] Cen, L.-X., Li, X. Q., Shao, J., Yan, Y. J.: Quantifying quantum discord and entanglement of formation via unified purifications. Phys. Rev. A 83, 054101 (2011)

[41] Adesso, G., Datta, A.: Quantum versus Classical Correlations in Gaussian States. Phys. Rev. Lett. 105, 030501 (2010); Giorda, P., Paris, M., G., A.: Gaussian Quantum Discord. ibid. 105, $020503(2010)$

[42] Ali, M., Rau, A.R.P., Alber, G.: Quantum discord for two-qubit X states. Phys. Rev. A 81, 
042105 (2010); see also Ali M. , Rau, A., R., P., Alber, G., ibid. 82, 069902(E) (2010)

[43] Shi, M., Yang, W., Jiang, F., Du, J.: Quantum discord of two-qubit rank-2 states. J. Phys. A: Math. Theor. 44, 415304 (2011)

[44] Chen, Q., Zhang, C., Yu, S., Yi, X., X., Oh, C., H.: Quantum discord of two-qubit X states. Phys. Rev. A 84, 042313 (2011)

[45] Lu, X., M., Ma, J., Xi, Z., Wang, X.: Optimal measurements to access classical correlations of two-qubit states. Phys. Rev. A 83, 012327 (2011)

[46] Girolami, D., Adesso, G.: Quantum discord for general two-qubit states: Analytical progress. Phys. Rev. A 83, 052108 (2011)

[47] Li, B., Wang, Z., X., Fei, S., M.: Quantum discord and geometry for a class of two-qubit states. Phys. Rev. A 83, 022321 (2011)

[48] Huang, Y.: Computing quantum discord is NP-complete. New J. Phys., 16, 033027 (2014)

[49] Dakić, B., Vedral, V., Brukner, Č.: Necessary and Sufficient Condition for Nonzero Quantum Discord. Phys. Rev. Lett. 105, 190502 (2010)

[50] Gessner, M., Laine, E.-M., Breuer, H.-P., Piilo, J.: Correlations in quantum states and the local creation of quantum discord. Phys. Rev. A 85, 052122 (2012)

[51] Modi, K., Brodutch, A., Cable, H., Paterek, T., Vedral, V.: The classical-quantum boundary for correlations: Discord and related measures. Rev. Mod. Phys. 84, 1655 (2012)

[52] Luo, S.: Using measurement-induced disturbance to characterize correlations as classical or quantum. Phys. Rev. A 77, 022301 (2008)

[53] Modi, K., Vedral, V.: Unification of quantum and classical correlations and quantumness measures. AIP Conf. Proc. 1384, 69 (2011)

[54] Brodutch, A., Modi, K.: Criteria for measures of quantum correlations. Quantum Inf. Comput. 12, 721-742 (2012)

[55] Adesso, G., Bromley, T.R., Cianciaruso, M.: Measures and applications of quantum correlations. J. Phys. A: Math. Theor. 49, 473001 (2016).

[56] Nielsen, M.A., Chuang, I.L.: Quantum Computation and Quantum Information (Cambridge University Press, Cambridge, England, 2000); Hayashi, M., Quantum Information: An Introduction (Springer-Verlag, Berlin, 2006)

[57] Bengtsson, I., Zyczkowski, K.: Geometry of Quantum States: An Introduction to Quantum Entanglement. Cambridge University Press (2006) 
[58] Uhlmann, A.: The transition probability in the state space of a $\star$-algebra. Rep. Math. Phys. 9, $273(1976)$

[59] Jozsa, R.: Fidelity for Mixed Quantum States. J. Mod. Opt 41, 2315 (1994)

[60] Bures, D., J., C.: An extension of Kakutani's theorem on infinite product measures to the tensor product of semifinite $\mathrm{W}^{*}$-algebras. Trans. Am. Math. Soc. 135, 199 (1969)

[61] Dodonov, V., V., Man'ko, O., V., Man'ko, V., I., Wunsche, A.: Hilbert-Schmidt distance and non-classicality of states in quantum optics. J. Mod. Opt. 47, 633 (2000)

[62] Luo, S., Zhang, Q.: Informational distance on quantum-state space. Phys. Rev. A 69, 032106 (2004)

[63] Majtey, A., P., Lamberti, P., W., Prato, D., P.: Jensen-Shannon divergence as a measure of distinguishability between mixed quantum states. Phys. Rev. A 72, 052310 (2005)

[64] Spehner, D., Illuminati, F., Orszag, M., Roga, W.: Geometric Measures of Quantum Correlations with Bures and Hellinger Distances. In: Fanchini F., Soares Pinto D., Adesso G. (eds) Lectures on General Quantum Correlations and their Applications. Quantum Science and Technology. Springer, Cham (2017) I 